\title{
Uma análise de investimentos considerando fatores intangíveis
}

\author{
Leila Keiko Canegusuco Jansen (POLIUSP) leila.jansen@poli.usp.br \\ Tamio Shimizu (POLIUSP) tmshimiz@usp.br \\ José Ulisses Jansen (IPEN) nana021295@uol.com.br
}

\begin{abstract}
Resumo
As análises para investimentos nas organizações ganham em amplitude quando se utilizam ferramentas de suporte à tomada de decisão, algumas de fácil utilização inclusive sem o uso de softwares especificos, que permitem aos decisores acrescentarem fatores intangiveis aos tradicionais critérios econômicos na avaliação de melhores alternativas. Este artigo apresenta um estudo de caso em uma empresa de médio porte, aqui denominada Alfa, do setor eletro-eletrônico, para escolha de nova fonte de energia térmica. Na escolha do investimento também foram acrescentados critérios qualitativos: impacto ambiental $e$ facilidade de operação. A avaliação das alternativas foi feita por dois métodos, o da Teoria da Utilidade e o Analytic Hierarchy Process -AHP, que permitem o tratamento de múltiplos critérios e a inserção de variáveis qualitativas. Apenas para as duas melhores alternativas a análise foi complementada com uma comparação financeira dos custos operacionais futuros. Conclui-se que as metodologias de tomada de decisão possibilitam considerar de modo consistente importantes elementos intangiveis na decisão de investimentos industriais.

Palavras-chave: Análise de investimento, Decisão, Teoria da Utilidade, Analytic Hierarchy Process.
\end{abstract}

\section{Introdução}

Tradicionalmente as empresas elaboram estudos de viabilidade técnica e econômica para avaliarem alternativas e decidirem pelos seus investimentos. Entretanto, em certos casos, existem critérios intangíveis de natureza diversa tais como a ambiental, social, política, de mercado, que são por vezes deixados de lado dadas as dificuldades em quantificá-los, mas que pela sua importância deveriam compor a lista de fatores a considerar.

O impacto ambiental oriundo das implantações industriais é uma questão que cresceu em relevância mundialmente nas últimas décadas. A geração de produtos para desenvolver a economia faz com que se interfira diretamente no ambiente através da subtração dos seus recursos ou da eliminação de componentes que o contaminam e degradam. O impacto ambiental das atividades que desequilibram o ambiente é não raramente difícil de ser quantificado, mas deve ser considerado nas análises de investimentos.

A facilidade de operação dos processos também é um outro fator intangível que deve ser levado em conta na definição dos investimentos industriais. Ela tem efeito direto no andamento correto dos processos e na motivação dos funcionários que por sua vez influenciam na qualidade dos produtos.

Neste artigo será apresentado um estudo de caso realizado em uma empresa de médio porte do setor eletro-eletrônico situada no estado de Minas Gerais. O objetivo da empresa era escolher uma nova fonte de energia térmica para o setor de utilidades da planta e os dois critérios qualitativos anteriormente citados foram inseridos na avaliação.

Trata-se de um problema de decisão sem risco e com múltiplos critérios. Para o estudo foram escolhidos dois métodos de apoio à decisão: o método da Teoria da Utilidade associado a cada um dos critérios - de uso bastante disseminado, e o método AHP - pela facilidade de ter o software. Foram avaliadas seis alternativas de implantação segundo cinco critérios definidos.

Dada a quantidade dos critérios utilizados, por praticidade, a análise de sensibilidade foi substituída por uma comparação financeira dos custos operacionais futuros das duas melhores alternativas. 


\section{Métodos para a escolha da melhor decisão}

Normalmente o processo de tomada de decisão envolve múltiplos critérios (também denominados de objetivos ou fatores) e variáveis qualitativas. Os métodos clássicos de otimização da programação matemática não estão bem definidos para resolver tais problemas, que podem, entretanto, ser tratados pela maioria dos métodos da Teoria da Decisão.

Entre os métodos usados para a escolha da melhor decisão cita-se:

- Teoria da Utilidade. É um método muito antigo, mas ainda muito utilizado que assume que o decisor deseja fazer uma escolha que corresponda ao maior nível de satisfação ou utilidade. As preferências do decisor são representadas por funções matemáticas chamadas de função utilidade. Esse método resolve algumas limitações da teoria da probabilidade (Paradoxo de S.Petersburgo), mas tem seu alcance contestado (Contra exemplo de Allais).

- Elimination et Choix Traduisant la Realité - ELECTRE. Trata-se de um algoritmo que reduz o tamanho do conjunto de alternativas possíveis por sua classificação de acordo com o critério da dominância de uma alternativa sobre outra. Um método semelhante é o Promethee.

- Analytic Hierarchy Process - AHP. Foi criado por Saaty na década de 70. A estruturação do problema começa na definição de um objetivo global. A seguir definem-se os subobjetivos numa estrutura de árvore, tendo como raiz o objetivo global. Conforme se afastam da raiz os fatores se tornam mais específicos. As extremidades, as folhas da árvore, representam os critérios ou objetivos. O ANP é uma extensão deste método.

- Measuring Attractiveness by a Categorical Based Evaluation Technique - MACBETH. Este método critica o uso de funções com valor cardinal. Ele fornece um indicador de inconsistência do conjunto de critérios formulados o que facilita sua revisão por Programação Linear.

- Treats, Opportunities, Weakness and Strengths - TOWS. O método relaciona e analisa os fatores internos e ambientais. Assemelha-se aos Fatores Críticos de Sucesso de Rockart.

\section{Método da Teoria da Utilidade associada a cada um dos critérios}

Os problemas de decisão que envolvem mais de um critério ou objetivo podem ser resolvidos quando os valores de cada critério são transformados para uma mesma unidade de medida. Assim, objetivos como valor do ganho esperado, vantagem competitiva, distância, podem ser transformados para uma mesma unidade de medida chamada de utilidade, com valores variando entre 0 e 1 .

A operacionalização do método da Teoria da Utilidade segue os seguintes passos:

- Estruturação do problema em uma Matriz de Decisão, que é uma tabela onde são lançados nas colunas os elementos do problema como cenários, critérios, probabilidades, alternativas de decisão;

- Construção da Árvore de Decisão, que é uma representação clara e muito útil quando o problema apresenta múltiplas variáveis, múltiplos objetivos e etapas de decisão. Cada ramo da árvore é formado por uma alternativa de decisão contendo os cenários, as probabilidades, as variáveis e os objetivos.

- Conversão dos valores dos critérios em valores da utilidade. Os valores variam entre 0 e 1 . Atribui-se o valor 0 ao pior valor e o valor 1 ao melhor valor. Os valores intermediários podem ser alocados segundo uma reta (neutra ao risco) ou curvas (aversão ao risco ou preferência pelo risco).

- Atribuição de pesos relativos a cada objetivo. Cada um dos objetivos recebe um peso relativo à sua importância. Ao objetivo mais importante é atribuído o valor 1; os demais objetivos recebem seus pesos relativamente ao objetivo de maior importância. 
- Normalização dos pesos dos objetivos fazendo com que a soma dê 1 .

- Cálculo da média ponderada dos objetivos para cada uma das alternativas fornecendo o valor da utilidade média.

- A alternativa a ser escolhida será a que apresentar o maior valor de utilidade média.

- Análise da sensibilidade. Como a atribuição dos pesos afeta o resultado da decisão é interessante analisar como a variação dos pesos afeta o resultado da decisão.

\section{Método Analytic Hierarchy Process - AHP}

O Analytic Hierarchy Process- AHP tem como um dos objetivos representar os modelos de modo mais realista incluindo todas as medidas importantes tangíveis ou intangíveis, fatores quantitativamente mensuráveis ou qualitativos (SAATY, 1980).

O AHP é um dos métodos mais conhecidos e utilizados podendo ser empregado em diversas situações como avaliação de custos e benefícios, alocação de recursos, medida de desempenho (benchmarking), pesquisa de mercado, determinação de requisitos, decisões estratégicas, negociação e resolução de conflitos, entre outras.

O método consiste das quatro etapas básicas que se seguem (COOK e RUSSEL, 1993):

- Desenvolvimento dos níveis de hierarquia de decisão dos elementos inter-relacionados.

- Determinação de preferências através de comparações paritárias.

- Síntese e determinação de prioridade relativa ou peso de cada elemento de decisão em um dado nível usando o método do auto-valor ou outro método de aproximação.

- Agregação das prioridades relativas para a escolha final.

A escala de comparações paritárias para o AHP é a apresentada no Quadro 1:

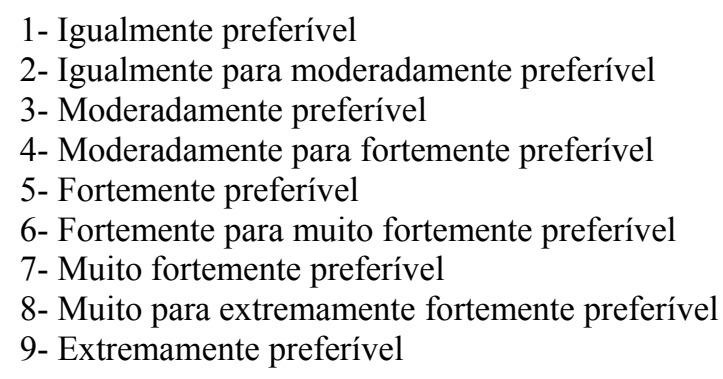

Quadro 1 - Escala de comparações paritárias para o AHP.

\section{Estudo de Caso}

Neste item é relatado um trabalho prático onde se procurou através do uso de métodos de apoio à decisão, confirmar a escolha da direção de uma empresa, que será denominada no artigo como empresa Alfa, para a realização de investimento em unidade de energia térmica. Todo o processo de análise das alternativas havia até então sido baseado em análise de viabilidade técnica e econômica, como é comum no caso de investimentos industriais. Havia, entretanto, a intenção dos decisores de inserir na avaliação dois fatores qualitativos: o impacto ambiental que a implantação causaria e a facilidade de operação da instalação, significando a busca por um processo que não apresentasse interrupções constantes para manutenção que sempre significam diminuição na qualidade dos produtos.

Inicialmente se utilizou o método da Teoria da Utilidade associada a cada um dos critérios. $\mathrm{O}$ empate de alternativas como melhor alternativa incentivou o uso do outro método, o AHP. 
O problema pode ser definido do seguinte modo: A empresa Alfa necessita de nova fonte de energia térmica com capacidade para $600 \mathrm{kcal} / \mathrm{h}$ para seu setor de utilidades.

Entre as alternativas totalmente disponíveis na região, no estado de Minas Gerais, encontramse: o uso de instalação à base de óleo diesel conjugado à energia elétrica - que seria uma ampliação da instalação existente; o BPF 1A; o BPF 2A e o GLP (gás liquefeito de petróleo). A empresa, entretanto, quis acrescentar na análise duas outras alternativas para efeito de comparação: o GN (gás natural) - que estará disponível dentro de quatro anos; e o GNL (gás natural liquefeito)- que ainda não foi utilizado por nenhuma indústria na região.

Compõem os critérios de avaliação os seguintes fatores: Investimentos adicionais - são as instalações complementares para a instalação da fonte de energia; Preço do equipamento; Facilidade de operação ; Impacto ambiental e Custo de operação durante o primeiro ano.

A estruturação do problema em uma matriz de decisão apresentando todas as alternativas de escolha e os critérios definidos é apresentada na Tabela 1:

\begin{tabular}{|l|l|c|c|c|c|c|c|}
\hline \multicolumn{2}{|c|}{ Critérios } & \multicolumn{6}{c|}{ Alternativas energéticas } \\
\cline { 3 - 7 } \multicolumn{2}{|c|}{} & A1 & A2 & A3 & A4 & A5 & A6 \\
GLP & GNL & GN & BPF1/A & BPF2/A & Diesel/Elétrica \\
\hline C1 & Investimentos adicionais (R\$) & 25.000 & 25.000 & 25.000 & 100.000 & 100.000 & 10.000 \\
C2 & Preço do equipamento (R\$) & 75.572 & 75.572 & 75.572 & 97.000 & 97.000 & 0 \\
C3 & Facilidade de operação & Fácil & Fácil & Fácil & Difícil & Difícil & Regular \\
C4 & Impacto Ambiental & Baixo & Baixo & Baixo & Alto & Alto & Médio \\
C5 & Custo de operação 1o.ano(R\$) & 93.320 & 89.731 & 61.017 & 67.584 & 55.870 & 265.416 \\
\hline
\end{tabular}

Tabela 1- Matriz de decisão do problema da empresa Alfa.

\section{Resultados obtidos}

a. Utilizando o método da Teoria da Utilidade.

A árvore de decisão do problema é apresentada na Tabela 2:

\begin{tabular}{|c|c|c|c|c|c|}
\hline $\begin{array}{l}\text { Alternativas } \\
\text { energéticas }\end{array}$ & $\begin{array}{l}\text { Investimentos } \\
\text { Adicionais }\end{array}$ & $\begin{array}{l}\text { Preço do } \\
\text { equipamento }\end{array}$ & $\begin{array}{l}\text { Facilidade } \\
\text { de operação }\end{array}$ & $\begin{array}{l}\text { Impacto } \\
\text { ambiental }\end{array}$ & $\begin{array}{l}\text { Custo de } \\
\text { operação }\end{array}$ \\
\hline A1 & 25.000 & 75.572 & Fácil & Baixo & 93.320 \\
A2 & 25.000 & 75.572 & Fácil & Baixo & 89.731 \\
A3 & 25.000 & 75.572 & Fácil & Baixo & 61.017 \\
A4 & 100.000 & 97.000 & Difícil & Alto & 67.584 \\
A5 & 100.000 & 97.000 & Difícil & Alto & 55.870 \\
A6 & 10.000 & 0 & Regular & Médio & 265.416 \\
\hline
\end{tabular}

Tabela 2 - Árvore de decisão da empresa Alfa.

Para a definição dos valores das utilidade adotou-se na conversão uma preferência estritamente linear. Seguiu-se com a atribuição dos pesos relativos para cada critério e a normalização dos mesmos. Finalmente foram obtidos os valores das médias ponderadas para as alternativas. Todos esses dados estão apresentados na Tabela 3.

Pelo método da Teoria da Utilidade a melhor decisão é dada pela alternativa de maior média ponderada, que no problema em questão apresentou empate entre duas alternativas, ou seja, A3 e A6. E em segundo lugar, muito próximas das duas primeiras, se apresentaram também empatadas as alternativas A1 e A2. 


\begin{tabular}{|c|c|c|c|c|c|c|c|c|}
\hline \multirow[b]{2}{*}{ Critérios } & \multicolumn{2}{|c|}{ Pesos } & \multicolumn{6}{|c|}{ Alternativas energéticas } \\
\hline & Relativos & \begin{tabular}{|c|}
$\begin{array}{c}\text { Normali- } \\
\text { zados }\end{array}$ \\
\end{tabular} & $\begin{array}{c}\text { A1 } \\
\text { GLP } \\
\end{array}$ & $\begin{array}{r}\mathrm{A} 2 \\
\text { GNL } \\
\end{array}$ & $\begin{array}{l}\mathrm{A} 3 \\
\mathrm{GN} \\
\end{array}$ & $\begin{array}{c}\text { A4 } \\
\text { BPF1/A }\end{array}$ & $\begin{array}{c}\text { A5 } \\
\text { BPF2/A } \\
\end{array}$ & $\begin{array}{c}\text { A6 } \\
\text { Diesel/Elétrica }\end{array}$ \\
\hline Investimentos adicionais & 0,90 & 0,30 & 0,83 & 0,83 & 0,83 & 0,00 & 0,00 & 1,00 \\
\hline Preço do equipamento & 1,00 & 0,33 & 0,22 & 0,22 & 0,22 & 0,00 & 0,00 & 1,00 \\
\hline Facilidade de operação & 0,10 & 0,03 & 1,00 & 1,00 & 1,00 & 0,00 & 0,00 & 0,50 \\
\hline Impacto Ambiental & 0,10 & 0,03 & 1,00 & 1,00 & 1,00 & 0,00 & 0,00 & 0,50 \\
\hline Custo de operação & 0,90 & 0,30 & 0,82 & 0,84 & 0,94 & 0,98 & 1,00 & 0,00 \\
\hline Somas & 3,00 & 1,00 & & & & & & \\
\hline Médias Ponderadas & & & 0,64 & 0,64 & 0,67 & 0,29 & 0,30 & 0,67 \\
\hline
\end{tabular}

Tabela 3 - Médias ponderadas para as alternativas energéticas da empresa Alfa.

Chegado a este ponto, o método da Teoria da Utilidade pede que se faça uma análise de sensibilidade visto que a adoção dos pesos afeta o resultado da decisão. Entretanto, optou-se por utilizar também outro método de decisão, o AHP, para confirmar o resultado da decisão.

b. Utilizando o método AHP.

A Figura 1 apresenta o desenvolvimento dos níveis de hierarquia para a estruturação do problema pelo método AHP. Neste caso, todos os critérios quantitativos e qualitativos foram colocados num mesmo nível.

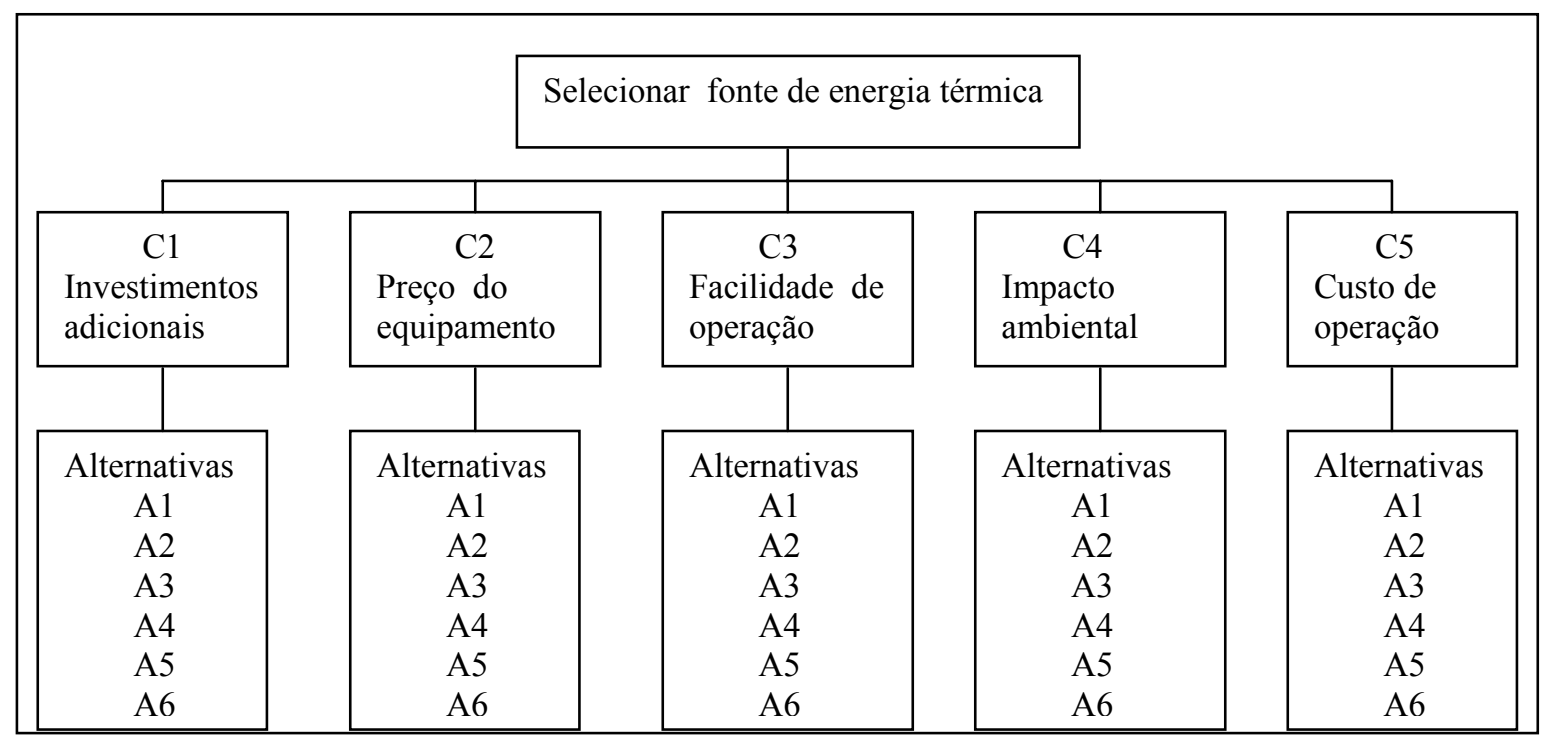

Figura 1 - Árvore de decisão hierárquica do problema da empresa Alfa.

A determinação das preferências através das comparações paritárias entres os critérios, seguindo a escala do Quadro 1 são apresentadas na Tabela 4.

\begin{tabular}{|l|c|c|c|c|c|}
\hline \multicolumn{1}{|c|}{ Critérios } & C1 & C2 & C3 & C4 & C5 \\
\hline C1- Investimentos adicionais & 1 & $1 / 2$ & 8 & 8 & 2 \\
C2- Preço do equipamento & 2 & 1 & 8 & 8 & 3 \\
C3- Facilidade de operação & $1 / 8$ & $1 / 8$ & 1 & 2 & $1 / 7$ \\
C4- Impacto Ambiental & $1 / 8$ & $1 / 8$ & $1 / 2$ & 1 & $1 / 7$ \\
C5- Custo de operação & $1 / 2$ & $1 / 3$ & 7 & 7 & 1 \\
\hline \multicolumn{1}{|c}{ totais } & 3,750 & 2,083 & 24,500 & 26,000 & 6,286 \\
\hline
\end{tabular}

Tabela 4 - Matriz de comparações paritárias entre os critérios do problema da empresa Alfa. 
A seguir foi determinada a prioridade relativa ou peso de cada critério. $O$ teste de consistência foi realizada utilizando-se a estimação do maior auto-valor da matriz paritária pela média aritmética dos elementos do vetor de consistências.

Do mesmo modo foi realizada a determinação das preferências através das comparações paritárias entre as alternativas para cada um dos critérios. Elas são apresentadas na Tabela 5:

\begin{tabular}{|c|c|c|c|c|c|c|}
\hline \multicolumn{7}{|c|}{$\begin{array}{l}\mathrm{C} 1 \text { - Investimentos } \\
\text { adicionais }\end{array}$} \\
\hline & $\mathrm{A} 1$ & $\mathrm{~A} 2$ & $\mathrm{~A} 3$ & A4 & A5 & A6 \\
\hline A1 & 1 & 1 & 1 & 8 & 8 & $1 / 3$ \\
\hline $\mathrm{A} 2$ & 1 & 1 & 1 & 8 & 8 & $1 / 3$ \\
\hline $\mathrm{A} 3$ & 1 & 1 & 1 & 8 & 8 & $1 / 3$ \\
\hline A4 & $1 / 8$ & $1 / 8$ & $1 / 8$ & 1 & 1 & $1 / 9$ \\
\hline A5 & $1 / 8$ & $1 / 8$ & $1 / 8$ & 1 & 1 & $1 / 9$ \\
\hline A6 & 3 & 3 & 3 & 9 & 9 & 1 \\
\hline
\end{tabular}

\begin{tabular}{|c|c|c|c|c|c|c|}
\hline \multicolumn{7}{|c|}{ C2 - Preço do equipamento } \\
\hline & A1 & A2 & A3 & A4 & A5 & A6 \\
\hline A1 & 1 & 1 & 1 & 4 & 4 & $1 / 7$ \\
\hline A2 & 1 & 1 & 1 & 4 & 4 & $1 / 7$ \\
\hline A3 & 1 & 1 & 1 & 4 & 4 & $1 / 7$ \\
\hline A4 & $1 / 4$ & $1 / 4$ & $1 / 4$ & 1 & 1 & $1 / 9$ \\
\hline A5 & $1 / 4$ & $1 / 4$ & $1 / 4$ & 1 & 1 & $1 / 9$ \\
\hline A6 & 7 & 7 & 7 & 9 & 9 & 1 \\
\hline
\end{tabular}

\begin{tabular}{|c|c|c|c|c|c|c|}
\hline \multicolumn{7}{|c|}{ C3 - Facilidade de Operação } \\
\hline & A1 & A2 & A3 & A4 & A5 & A6 \\
\hline A1 & 1 & 1 & 1 & 9 & 9 & 6 \\
\hline A2 & 1 & 1 & 1 & 9 & 9 & 6 \\
\hline A3 & 1 & 1 & 1 & 9 & 9 & 6 \\
\hline A4 & $1 / 9$ & $1 / 9$ & $1 / 9$ & 1 & 1 & $1 / 6$ \\
\hline A5 & $1 / 9$ & $1 / 9$ & $1 / 9$ & 1 & 1 & $1 / 6$ \\
\hline A6 & $1 / 6$ & $1 / 6$ & $1 / 6$ & 6 & 6 & 1 \\
\hline
\end{tabular}

\begin{tabular}{|c|c|c|c|c|c|c|}
\hline \multicolumn{7}{|c|}{ C4 - Impacto ambiental } \\
\hline & A1 & A2 & A3 & A4 & A5 & A6 \\
\hline A1 & 1 & 1 & 1 & 9 & 9 & 6 \\
\hline A2 & 1 & 1 & 1 & 9 & 9 & 6 \\
\hline A3 & 1 & 1 & 1 & 9 & 9 & 6 \\
\hline A4 & $1 / 9$ & $1 / 9$ & $1 / 9$ & 1 & 1 & $1 / 6$ \\
\hline A5 & $1 / 9$ & $1 / 9$ & $1 / 9$ & 1 & 1 & $1 / 6$ \\
\hline A6 & $1 / 6$ & $1 / 6$ & $1 / 6$ & 6 & 6 & 1 \\
\hline
\end{tabular}

\begin{tabular}{|c|c|c|c|c|c|c|}
\hline C5 - Custo de operação \\
\hline & A1 & A2 & A3 & A4 & A5 & A6 \\
\hline A1 & 1 & 1 & $1 / 3$ & $1 / 2$ & $1 / 3$ & 7 \\
\hline A2 & 1 & 1 & $1 / 3$ & $1 / 2$ & $1 / 3$ & 7 \\
\hline A3 & 3 & 3 & 1 & 2 & 1 & 9 \\
\hline A4 & 2 & 2 & $1 / 2$ & 1 & $1 / 2$ & 8 \\
\hline A5 & 3 & 3 & 1 & 2 & 1 & 9 \\
\hline A6 & $1 / 7$ & $1 / 7$ & $1 / 9$ & $1 / 8$ & $1 / 9$ & 1 \\
\hline
\end{tabular}

Tabela 5 - Matrizes de comparações paritárias entre alternativas para cada critério do problema da empresa Alfa.

Finalmente, as prioridades relativas de todos os elementos da decisão e a prioridade composta são apresentadas na Tabela 6:

\begin{tabular}{|c|c|c|c|c|c|c|c|}
\hline \multicolumn{8}{|c|}{ rioridade composta para as alternativas A1 A2 A3 A4 A5 e A6 } \\
\hline & $\mathrm{C} 1$ & $\mathrm{C} 2$ & $\mathrm{C} 3$ & $\mathrm{C} 4$ & $\mathrm{C} 5$ & Prioridade relativa & Prioridade composta \\
\hline A1 & 0,181 & 0,12 & 0,285 & 0,285 & 0,109 & 0,292 & 0,149 \\
\hline$\overline{\mathrm{A} 2}$ & 0,181 & 0,12 & 0,285 & 0,285 & 0,109 & 0,425 & 0,149 \\
\hline A3 & 0,181 & 0,12 & 0,285 & 0,285 & 0,291 & 0,047 & 0,186 \\
\hline $\mathrm{A} 4$ & 0,028 & 0,038 & 0,027 & 0,027 & 0,176 & 0,035 & 0,062 \\
\hline A5 & 0,028 & 0,038 & 0,027 & 0,027 & 0,291 & 0,201 & 0,085 \\
\hline$\overline{\mathrm{A} 6}$ & 0,401 & 0,565 & 0,09 & 0,09 & 0,023 & & 0,369 \\
\hline
\end{tabular}

Tabela 6 - Prioridade composta para as alternativas 
Pelo método AHP a melhor decisão é dada pela alternativa de maior valor da prioridade composta. No caso em questão é a alternativa A6. Em segundo lugar encontra-se a alternativa A3, seguida pela A1 e A2.

\section{Comparação econômica entre as duas melhores alternativas}

De posse dos resultados dos dois métodos utilizados para escolha da melhor decisão e sabendo-se que ambos os métodos utilizam atribuições de pesos, um estudo de sensibilidade deveria ser realizado. Mas, por praticidade se resolveu substituir a análise de sensibilidade por uma comparação do custo de operação futuro para as duas melhores alternativas, visto que uma das informações fornecidas pela direção da empresa Alfa é de que é muito importante minimizar o fluxo de caixa.

A alternativa A6, diesel conjugado à elétrica, foi a primeira colocada em ambos os métodos. Ela foi, portanto, uma das escolhidas para a comparação econômica.

A alternativa A3, gás natural, também foi a primeira colocada em um método e a segunda em outro. Mas, a alternativa A3 representa o uso do gás natural, que estará disponível apenas daqui a quatro anos, o que a descartará neste momento da comparação.

As alternativas A1 e A2 vêm a seguir na ordem de preferência. A alternativa A2, o gás natural liquefeito ainda não foi utilizado por nenhuma indústria na região, o que o cerca de dúvidas técnicas. Resta portanto a alternativa A1.

Uma análise comparativa dos custos de operação futuros foi realizada entre as alternativa A1 e A6.

Os dados utilizados para a comparação encontram-se na Tabela 7 :

\begin{tabular}{|c|c|c|}
\hline Alternativa & Investimento inicial $(\mathrm{R} \$)$ & Custo operacional (R\$/mês) \\
\hline $\mathrm{A} 1$ & $100.572,00$ & $7.777,00$ \\
$\mathrm{~A} 6$ & $10.000,00$ & $22.118,00$ \\
\hline
\end{tabular}

A comparação das duas alternativas procurou vislumbrar qual seria o impacto que cada uma delas representaria mensalmente no fluxo de caixa da empresa. Para os cálculos mensais foi utilizada uma taxa de juros de $2 \%$ ao mês. A alternativa A1 indica ser a mais adequada considerando-se os custos operacionais ao longo dos meses. O resultado pode ser visualizado no gráfico apresentado na Figura 2.

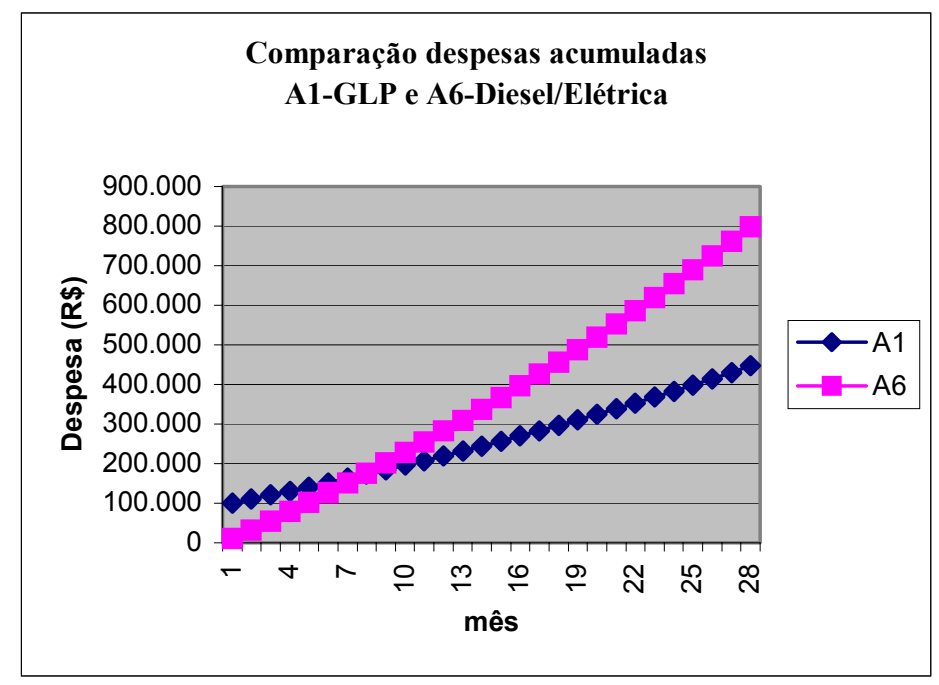

Figura 2 - Comparação das despesas acumuladas ao longo dos meses para as alternativas A1 e A6 do problema da empresa Alfa. 


\section{Conclusões}

O problema real apresentado de escolha entre várias alternativas para um dado investimento é um exemplo bastante comum no dia a dia das empresas. Entretanto, em muitos casos os fatores intangíveis pertinentes ao problema nem sempre são considerados. Este artigo mostrou que a introdução de elementos tais como impacto ambiental e facilidade de operação, que apesar de não terem sido apreciados como de alto peso diante de outros elementos, podem ser considerados de modo consistente em uma decisão.

$\mathrm{O}$ artigo demonstrou também que alguns métodos de suporte à tomada de decisão, como a Teoria da Utilidade e AHP podem ser facilmente utilizados. O método da Teoria da Utilidade possui uma limitação, apresentada no momento de se realizar a análise de sensibilidade quando existem muitos critérios. Esta dificuldade pôde, entretanto, ser ultrapassada por uma simples análise econômica comparativa entre as duas melhores alternativas.

\section{Referências}

COOK, T.M.\& RUSSEL, R.A. (1993) - Introduction to Management Science. Prentice Hall. New Jersey.

SAATY, T.L. (1980) - The Analytic Hierarchy Process. McGraw-Hill. New York. 